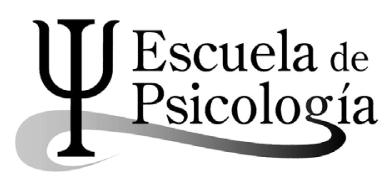

Wímb lu, Rev. electrónica de estudiantes Esc. de psicología, Univ. de Costa Rica. 9(1): 29-42, 2014 / ISSN: 1659-2107

\title{
LA MATERNIDAD COMO UN CONSTRUCTO SOCIAL DETERMINANTE EN EL ROL DE LA FEMINIDAD
}

Motherhood as a determinant role in the social construction of femininity

\author{
Karla Barrantes Valverde ${ }^{l}$ \\ María Fernanda Cubero Cubero ${ }^{2}$
}

\begin{abstract}
Resumen: El presente artículo presenta una revisión bibliográfica basada en el tema de la concepción de maternidad en diferentes momentos históricos, y cómo esta ha sido asociada directamente con la feminidad. Lo anterior, se presentará a través de una recopilación de información de cómo se vivió y vive actualmente la maternidad por parte de las mujeres, es así como se iniciara desde la sociedad primitiva, sociedad matriarcal, inicio de la sociedad patriarcal, época renacentista y Revolución Industrial, para llegar a lo que desde el feminismo se expone sobre el tema y algunas consideraciones de cómo se vive en la actualidad.
\end{abstract}

Palabras clave: maternidad, constructo social, mujer, feminidad, género.

Abstract: The present article contains a bibliographical revision which objective is to investigate the concept of motherhood in different historical moments and how it has been automatically associated with femininity of women. This will be presented through a compilation of information of how lived and lives motherhood by women, is also was started from primitive society, matriarchal society, patriarchal society, Renaissance and Industrial Revolution, to reach what is exposed from feminism on the subject and some considerations of how this still living today.

Key Words: motherhood, social construct, woman, feminity, gender.

1 Estudiante de licenciatura en psicología. Universidad de Costa Rica. Correo electrónico: karla_bts@hotmail.com

2 Estudiante de licenciatura en psicología. Universidad de Costa Rica. Correo electrónico: ferdadi@live.com

Recepción: 25/10/2013 Aceptación: 13/02/2014 


\section{Introducción}

La maternidad ha sido entendida como un elemento fundamental en la esencia femenina, lo cual ha provocado que se relacione la palabra mujer con el hecho de ser madre. Sin embargo, desde una posición feminista, esta postura ha sido debatida, ya que se considera inexistente la relación mencionada anteriormente, pues esta se entiende como una representación cultural (Marrades, 2002).

Además, se entiende que la maternidad es un término que se encuentra en permanente evolución y en el cual incide tanto factores culturales como sociales, los cuales lo han relacionado con las definiciones de mujer, procreación y crianza (Molina, 2006).

A partir de lo anterior, se deja en evidencia como el concepto de maternidad ha sido asociado con una característica propia de la mujer. Se debe mencionar que esta idea ha sido permeada por lo establecido cultural y socialmente, pues como lo menciona Royo (2011, p.28) la maternidad "es un constructo social y simbólico que adquiere diferentes significados en diferentes contextos sociohistóricos".

De esta manera, se plantea la presente investigación, en la cual se pretende considerar y analizar cómo los cambios que se han dado en la concepción de maternidad, a lo largo de la historia, han determinado el papel que las mujeres deben cumplir.

\section{Condición de la mujer en la sociedad matriarcal}

Según Beauvoir (2005), el comunismo primitivo estaba caracterizado por ofrecer las mismas condiciones a los hombres y mujeres, de manera que no existía diferencia entre las tareas que estos debían realizar, por lo que al tener que enfrentar una mujer el proceso de embarazo y maternidad, se daba una pequeña interrupción en las labores de caza y recolección de frutas que realizaba comúnmente con los demás integrantes de la tribu. Sin embargo, las diferencias entre sexos se empiezan a denotar con el surgimiento de la agricultura, descubrimiento que ha sido atribuido a la mujer y que reflejó un avance en la organización económica.

Las diferencias surgen también a raíz de la imposición de roles por la división del trabajo, la cual según Vélez (2007) atribuye a las mujeres las tareas relacionadas con la maternidad, siendo estas aprendidas desde el nacimiento del individuo al ser este parte de una cultura. Esta cultura es definida por Lewis (1985, p.41) como un "sistema integral de patrones de conducta aprendidos, característicos de los miembros de una sociedad", de manera que la maternidad como otras conductas son adquiridas a partir de que las mujeres son parte de una sociedad. 
De acuerdo con lo anterior, las tareas que debe realizar una persona están delegadas a partir del sexo de la misma, para lo cual influye lo impuesto culturalmente. Para el caso de esta investigación, es importante resaltar que la maternidad es un patrón de conducta a seguir que se le ha atribuido a toda mujer desde la sociedad primitiva, dándosele a dicho patrón de conducta características específicas según lo impuesto por la cultura, la sociedad y el momento histórico que se atraviesa. Con respecto a lo anterior se debe resaltar que dichas características son parte de los elementos que intervienen en la organización social.

En relación con ello, Walkowitz (1995) señala que fue esta labor asignada a las mujeres la que marcó las diferencias entre los sexos y con esto los trabajos que debían realizar los individuos, pues se tenía claro que la labor de la mujer iba a ser lo referente a la maternidad, excluyéndose a los hombres de esta tarea y otorgándosele otras obligaciones. Cabe destacar, que además de las diferencias entre las labores que realizan un hombre o una mujer, también se establecen características representativas para la mujer a partir del rol que asumen. Un ejemplo de ello es la característica de que las mujeres sean vistas cómo cuidadoras, siendo esto representativo del rol de madre y no característico de lo que debería hacer un hombre.

Retomando lo expuesto anteriormente, Aguirre (1989) agrega que en la división de trabajos se le atribuye al hombre la misión específica de la caza, mientras que a la mujer se le delega lo referente a la maternidad, considerándose a esta fuente de fecundidad y con esto de prosperidad. A partir de esta tarea otorgada a la mujer, Beauvoir (2005) indica que ella empieza a llevar a cabo labores como la artesanía y la agricultura, pues se consideraba que dichas actividades podían llevarse a cabo en conjunto con las que se les atribuía por ser madres.

A través de la invención de la agricultura la mujer alcanza un lugar importante en la sociedad, pues esta actividad representó un avance en la economía del grupo y el principal medio de producción que les permitía la sobrevivencia; lo cual coloca a la mujer en una posición de utilidad, que le daba poder y prestigio. De esta manera, se deja en evidencia que durante esta época se hace presente el matriarcado, en donde las femeninas eran la fuente de todo poder, según lo menciona Aguirre (1989).

En relación con el descubrimiento de la agricultura así como la importancia y demanda que esta cobró, la maternidad vino a cumplir una función importante para el desarrollo de dicha actividad, ya que para este período las mujeres debían procrear hijos, para que estos trabajaran con los diferentes cultivos. A partir de lo anterior surge la analogía de la mujer con la tierra, como dadoras de vida y fuentes de riqueza (Beauvoir, 2005). De esta manera, se evidencia un cambio con respecto a la concepción que se tenía anteriormente en relación con 
la maternidad, ya que para dicho período se resalta e incluso se venera esta condición, contrario a lo que sucedía durante el período de caza y recolección, en el cual la maternidad era irrelevante.

Asimismo, Engels (2001) agrega que en el periodo de la sociedad matriarcal es instaurado el derecho materno, el cual surge a partir de la presencia de familias por grupos, en las cuales debido a la poligamia y poliandria, son las mujeres las que pueden distinguir sus propios hijos, aunque ellas deban brindar cuidados maternales a todos los miembros de la tribu. Es así como dentro de este tipo de familia la única manera de establecer la descendencia es por la línea materna, siendo además la única que pudo indicar las relaciones de herencia, dando esto un gran valor al papel de la madre.

De esta manera, se muestra cómo la figura de la mujer cobra importancia a raíz de su función procreadora, la cual permite que esta se coloque en un lugar de prestigio y relevancia a nivel social. Es así como las mujeres durante este período se caracterizaban por tener dominio en el sistema social existente y por poseer la tarea de proteger a sus hijos, viéndose esto reflejado en la división del trabajo (Aguirre y Engels, 2001).

A partir de lo anterior, se deja en evidencia cómo desde la época primitiva se instauraron características al rol de madre que aún se mantienen, aunque aspectos como lo es el poder social que significaba ser madre y que tenían las mujeres se fueron cambiando, como se dará a conocer en el siguiente apartado.

\section{Paso a la sociedad patriarcal: implicaciones en la maternidad}

La posición privilegiada que venía ocupando la mujer a nivel económico y social, se fue debilitando a partir de la presencia de algunas tribus dedicadas al pastoreo. Beauvoir (2005) da a conocer que a las madres durante el desarrollo de este tipo de actividad, les correspondía la tarea de cuidar y domesticar el rebaño que poseía la tribu, mientras que la tarea del hombre consistía en la captura de animales para que estos fueran parte de su posesión. En este tipo de actividad, la mujer madre pasa a ser subordinada, pues el hombre pasa a ocupar el lugar principal dentro de la tribu, considerándose lo que ella realiza como un trabajo secundario.

No obstante, se debe señalar que las mujeres solteras si tenían la posibilidad de acompañar a los hombres a realizar la labor que a ellos les correspondía, de manera que es la condición de maternidad la que define que la mujer pase a realizar una labor secundaria (Beauvoir, 2005). Dicha situación, es un claro ejemplo de lo explicado por Levis (1985), el cual menciona que la organización social se da en función de la productividad que tengan las actividades llevadas a cabo por los miembros de la tribu, lo que explica por qué la mujer madre va a 
ocupar un lugar secundario en la sociedad, ya que su tarea se consideraba menos productiva que la que realizaba el hombre.

La ruptura que se comenzó a marcar durante el modo de vida pastoril, se vio aumentada a través de la aparición de nuevos oficios relacionados con la artesanía y el surgimiento de la propiedad privada, lo cual fue determinante para el inicio de la sociedad patriarcal. Se debe aclarar que la artesanía y el comercio, se convierten en las principales actividades productivas, colocando al trabajo campesino en un lugar de menos importancia en relación con estas, lo cual a su vez provocó que la mujer perdiera el papel de la principal productora de la sociedad (Beauvoir, 2005).

A raíz de dicha situación, el papel fundamental de la mujer pasa a ser la procreación, ignorando las demás labores que ellas realizaban. Es así como durante la Edad Media, se considera que la capacidad que tiene la mujer para procrear hijos y con esto garantizar la descendencia de la familia, resulta ser el hecho por lo que es valorada, es decir, la maternidad se convierte en lo que le otorga importancia a la mujer, de manera que la esterilidad era considerada punto de deshonra y motivo por el que un hombre se podía divorciar (Beauvoir, 2005).

Beauvoir (2005) agrega que una característica de ser madre durante la Edad Media es cuidar a sus hijos y ser un ejemplo para ellos, además de cumplir con sus deberes de esposa y ama de casa. De modo que el ideal de mujer que se poseía para esta época, correspondía a aquella que cumplía con los deseos del hombre, siendo esto característico de la sociedad patriarcal; y que tenía conocimientos en medicina, por lo cual posteriormente fueron clasificadas como brujas y por ello perseguidas. Cabe destacar que para este periodo según dicho autor, las mujeres no poseían derechos.

De esta manera, la maternidad se convirtió en la característica y actividad fundamental de las mujeres, la cual era necesaria para que se le otorgara algún tipo de valor. Es decir, la mujer no tenía valor como ser humano sino hasta cuando esta lograba ser madre, de modo que las mujeres incluso se preparaban para dicha condición y también le delegaban una gran importancia.

\section{La maternidad en la época del Renacimiento}

En la época renacentista la perspectiva hacia la maternidad se ve influenciada por el pensamiento humanista característico de esta época. Es así como según Romero (1998), cuando la mujer estaba embarazada se consideraba que ella se debía cuidar para proteger con esto a su hijo también, sin embargo se considera que esto no es únicamente responsabilidad de la madre, sino también del padre o esposo, el cual se debía mostrar atento ante las necesidades y cuidados de su compañera e hijo, así también debía evitarle preocupaciones y trabajos a las futuras madres. Lo anterior demuestra el cambio crucial que se presentó en esta 
época, pues hasta ese momento la labor de madre de proteger, cuidar, atender y educar a los hijos había sido delegada únicamente a las mujeres.

Es importante destacar que este valor que se le otorgaba tanto a las mujeres gestantes como a los infantes que aun no habían nacido, surge debido al valor que se le daba desde el humanismo al individuo (Romero, 1998). Sin embargo, según Lozano (2001), desde los discursos moralistas y teológicos se consideraba que la libertad y la sexualidad femenina estaban únicamente dirigidas hacia la maternidad, de manera que la Iglesia condenaba aquellas mujeres que realizaran practicas eróticas que fueran enfocadas al placer y no a la procreación, siendo esta última la que le daba valor al sexo femenino.

Estos discursos teológicos mencionados anteriormente encuentran relación con la literatura católica que determinaba una serie de deberes religiosos a las mujeres, entre los cuales se destacan la suavidad, la compasión, el amor maternal, cuidado de los enfermos, ancianos y pobres, y la educación religiosa de sus hijos (Lozano, 2001). Dichos deberes y lo mencionado en el párrafo anterior, denotan una vez más como la mujer ha sido asociada siempre con la condición de maternidad, impidiéndosele incluso el disfrute de su libertad sexual, y ligándola a características y actitudes correspondientes al cuidado y amor maternal.

Para finalizar con este periodo, es importante señalar que a partir del renacimiento se expone la importancia de establecer una buena relación afectiva entre madre e hijo, por lo cual se enfatizaba en que las madres criaran a sus hijos y en que ellas fueran las que los amamantaran (Romero, 1998). De manera que en este periodo, a pesar de que se le empiezan atribuir funciones paternales a los hombres, la mayor responsabilidad del cuidado de los hijos o hijas recaía en las mujeres.

\section{La maternidad durante la Revolución Industrial}

En el siglo XIX, la sociedad pensaba que el sitio que debía ocupar las mujeres era el hogar, dándose así un rechazo hacia el trabajo realizado por ellas. Sin embargo, a finales del siglo XIX y a inicios del siglo XX, se comenzó a dar una mayor aceptación por parte de la sociedad para que las mujeres comenzaran a trabajar fuera del hogar, cuando fuera necesario, como en el caso de familias pobres, donde las mujeres se encargaron de mantener económicamente a sus familias (Núñez y Contreras, 2002).

A partir de lo anterior, se muestra como entre el siglo XIX y siglo XX, se comienzan a presentar una serie de cambios en torno al género femenino, siendo uno de estos cambios la posibilidad de que las mujeres comenzaran a realizar trabajos fuera del hogar, con lo cual obtuvieron nuevas tareas y responsabilidades. El hecho de que las mujeres comenzaran a experimentar cambios en las tareas que realizaban, provocó también cambios con respecto a la maternidad, la cual 
estaba relacionada con las labores del hogar, ya que algunas mujeres comenzaron a dejar de lado estas actividades para insertarse en el mercado laboral debido a sus necesidades económicas, presentándose a su vez diferencias en el cuidado que le brindaban a sus hijos.

Es importante indicar que durante la Revolución Industrial, las mujeres trabajaban jornadas de diez e incluso doce horas, además ellas experimentaban otros abusos por parte de los patrones, ya que además recibían un muy mal pago por sus trabajos, siendo este aún menor que el dado a los varones, ya que a nivel social se daba una escasa valoración del trabajo femenino (Núñez y Contreras, 2002). Cabe destacar, que no contaban con el apoyo a nivel social para realizar trabajos fuera del hogar, pues se consideraba que la mujer debía estar en la casa realizando tareas domésticas, lo cual se relacionaba también con el cuidado de los niños.

Asimismo, Núñez y Contreras (2002) dan a conocer que durante la Revolución Industrial se realizaron señalamientos negativos con respecto al trabajo femenino, esto a partir del punto de vista de salud pública, apoyado por posturas científicas. Entre algunos de esos señalamientos estaba el presentar el trabajo femenino como un desencadenante de abortos, partos prematuros, entre otros.

Con respecto a lo anterior, se evidencia que los científicos establecieron una relación directa entre consecuencias negativas del trabajo femenino y la maternidad, tal y como lo indican Núñez y Contreras (2002). De esta manera, se deja en evidencia que incluso por medio de posturas científicas, se ejerció un rechazo hacia el trabajo realizado por las mujeres fuera de sus hogares, ya que se presentó dicho trabajo como un causante de problemas en el embarazo. A partir de lo anterior, se puede comprender que durante la Revolución Industrial existían grupos dentro de la sociedad que incentivaban la idea de que las mujeres se limitaran a las tareas del hogar y con esto al cuidado de los niños.

Además, se debe resaltar que al establecerse una relación directa entre trabajo femenino realizado fuera del hogar y problemas en el embarazo, se evidencia como por medio de la ciencia se intentó señalar aspectos negativos de dicho trabajo con respecto a las consecuencias perjudiciales que provocaba en el embarazo. Dichos planteamientos científicos tuvieron una incidencia en la sociedad con respecto a las ideas que se poseían en torno a la maternidad y el ser mujer, ya que algunas mujeres podían pensar en la posibilidad de no trabajar para evitar problemas en su embarazo. Otras mujeres podrían pensar que necesitaban trabajar para mantener económicamente a sus familias, esto a pesar de que se encontraran embarazadas, entre otras posibles pensamientos o cuestionamientos que se pudieron dar.

Núñez y Contreras (2002) dan a conocer que por parte de grupos comunistas se tenía la percepción de que la maternidad era una función específica de la mujer, por lo que se resaltaba la importancia de que el sexo femenino recibiera 
la protección necesaria en su trabajo para que la maternidad no se viera afectada de ninguna manera. A partir de esto, se muestra como por medio de estos grupos se comenzó a dar una cierta apertura para que las mujeres trabajaran fuera de sus hogares, siempre y cuando existiera una normativa laboral que protegiera a las mujeres como madres; lo cual fue un indicio para que en la actualidad las mujeres cuenten con leyes que las respalden en su trabajo y permitan también la protección de estas y de sus hijos.

Es importante señalar que a pesar de esa cierta apertura para que la mujer trabaje fuera del hogar, se seguía manteniendo la idea de que las mujeres son exclusivamente las responsables de la maternidad. De esta manera, se entiende que en el período de la Revolución Industrial se dieron cambios en las labores que realizaban las mujeres, lo cual generó ciertas variaciones en cómo las mujeres asumían la maternidad.

\section{Feminismo: un cambio drástico en la concepción de maternidad}

El movimiento feminista que surge entre los años 1960 y 1970, según Everingham (1997), tenía como objetivo principal la igualdad y la autonomía de la mujer, considerándose que la causa de no poseer dichas condiciones era el rol en la maternidad que las mujeres cumplían, pues debido a la crianza de los hijos se les dificultaba acceder a trabajos y se les colocaba en una posición inferior, de modo que resultaba necesario modificar el rol de madre que se venía presentando comúnmente, para lograr un cambio en la condición de la mujer. Es así como, el feminismo buscaba romper con las definiciones tradicionales de lo que era ser mujer, las cuales hacían una asociación automática de esta con la maternidad, condición que la delegaba en una posición de inferioridad y de sometimiento a los hombres, siendo esto un impedimento para alcanzar la autonomía por la cual luchaba el feminismo.

Por su parte, Gonzales (2010) señala que el feminismo se encuentra constituido por dos elementos, siendo uno de estos la visión de superar el patriarcado y promover la equidad de género. Dichas luchas suponían la igualdad de condiciones para las mujeres y la autonomía de estas, para lo cual también se considera necesario el empoderamiento de la mujer, el cual hace referencia a la posibilidad que tienen las mujeres para tomar decisiones, sentirse segura y poseer por ella misma una visión hacia el futuro, de manera que fueran ellas las que empezaran a definir y a decidir sobre este y sus acciones, entre las cuales se destaca la decisión de ser madres, la cual años atrás ni siquiera era considerada dentro de lo que una mujer podía elegir, pues el hecho de ser mujer hacía referencia directamente a ser mamá, lo cual se convirtió en una de las principales luchas para el movimiento emergente. 
En relación con el tema de la maternidad, el feminismo considera que la decisión de realizar un aborto o no, es únicamente de las mujeres, por lo cual una de sus principales luchas ha sido dirigida a la despenalización del mismo. Esta lucha nace debido a la situación en muchos países, en los cuales en el marco legal se penaliza la realización de un aborto que no sea de tipo terapéutico, llegándose a esta situación únicamente cuando la madre tiene en peligro su vida o en algunos países cuando el embarazo fue producto de una violación o un incesto (Gonzales, 2010). Lo anterior refleja un cambio en el camino que se venía siguiendo en torno a la maternidad, pues de ser una característica intrínseca a la mujer, se pasa a evaluar la posibilidad de que esta pueda abortar, lo cual representa un viraje en lo que venía sucediendo.

El feminismo fundamenta esta lucha considerando que el peligro para la vida de las mujeres se encuentra en la ilegibilidad del aborto, pues la muerte de estas por causa de esta práctica han sido considerables, para lo cual dicho movimiento apunta a la necesidad de que se legalice y que sean las mismas mujeres quienes decidan sobre su propio cuerpo y futuro (Gonzales, 2010), reflejándose de esta manera el empoderamiento que puede tomar la mujer para decidir si continua con un embarazo o no, lo cual a su vez demostraría el avance que se ha tenido con respecto al tema, pues ahora tendrían las mujeres la posibilidad de elegir en lo que había sido naturalizado por muchos años, lo cual incluso era lo que le daba valor a la mujer.

Gonzales (2010), menciona que el feminismo además de la lucha por la legalización del aborto, también establece una disputa para lograr la prevención de los embarazos no deseados, para lo cual es necesario una mayor apertura en el uso de anticonceptivos, así como un mayor acceso a la educación sexual integral, placentera y responsable, en la cual el empoderamiento de la mujer refleje la posibilidad de que puedan decidir sobre su cuerpo y protegerlo, además de su propio futuro.

En relación con lo anterior, se debe destacar el acontecimiento en la historia de la revolución anticonceptiva, la cual según Gándara y Puigvert (2005) sucedió en el siglo $\mathrm{XX}$, siendo esta un hecho que colaboró en gran manera en el apropiamiento de la identidad por parte de las mujeres, ya que a través de esto han tomado el control en su vida sexual. Lo anterior, denota un gran avance en la situación de las mujeres según Gonzales (2010), pues esto indicó la separación de la procreación y la sexualidad por medio de la utilización anticonceptivos.

Es así como la revolución anticonceptiva también fue un hecho que marcó la diferencia en la relación entre mujeres y maternidad, ya que a partir de la utilización de anticonceptivos las mujeres empiezan a decidir la posibilidad de ser madres o no serlo, es decir, la situación que anteriormente era ligada con la feminidad, a partir de esta revolución era una elección para las mujeres, ya que 
estas ahora empezaban a contar con recursos para limitar las posibilidades de llegar a ser madres.

\section{Maternidad en la actualidad}

La maternidad cambia con respecto a la época, por ello en la actualidad se hacen presentes una serie de cambios tales como que las mujeres reflexionan acerca de su propia vida, definen por sí mismas las oportunidades, peligros y prácticas con respecto a la maternidad, además se encargan de dar forma y organizar los vínculos con sus hijos, así como decidir acerca de su crianza. De esta manera, estas dan dirección a las experiencias relacionadas con la maternidad a pesar de que esta se encuentra bajo la influencia social, política y económica (Castillo, 2008).

El contexto histórico y el sistema social en el cual se encuentre la madre es determinante para las características que esta asume desde ese rol, es por ello que la entrada de la mujer en el mercado laboral implicó transformaciones en las actividades y actitudes que estas tenían, lo cual a su vez tuvo incidencia en cómo se asume lo referente a la maternidad. De este modo, para nuestros días las mujeres pueden tomar decisiones con respecto a la maternidad, como lo es decidir si desea tener hijos o no, o elegir cuantos hijos desea tener; a pesar de ser influidas y responder a los requerimientos del sistema vigente.

En relación con lo anterior, Castillo (2008) da a conocer que las mujeres comienzan a desenvolverse individualmente, como lo es por medio de una profesión, lo cual refleja no solo el deseo de superación, sino también la necesidad de poder responder a las exigencias que el sistema económico y social solicitan. Este cambio en la condición de la mujer relacionado con la inserción en el ámbito laboral tiene implicaciones en cómo se asume y se visualiza la maternidad, pues como lo menciona el autor, esta deja de ser excluyente y exclusiva de las mujeres, dándose también responsabilidad a los padres de sus hijos, lo que no sucedía años atrás.

En conjunto con estos cambios en la concepción de la maternidad, se presentan modificaciones en las labores domésticas que establecían que debían realizar las mujeres. Estos cambios también encuentran relación con el ingreso de las mujeres en la realización de labores fuera del hogar con un objetivo lucrativo, teniendo esto también incidencia en el hecho de que las madres deban solicitar ayuda a sus familiares o recurrir a instituciones de cuidado infantil para la educación y atención de sus hijos (Castillo, 2008), lo cual refleja un viraje en relación con las épocas mencionadas anteriormente, pues como se dio a conocer, en estas el cuidado de los niños era delegado únicamente a las madres, lo cual se convertía en la labor que debían realizar. 
Sin embargo, se debe considerar que las madres no dejan de preocuparse por el bienestar de sus hijos, por lo que debido a su situación recurren a distribuir su tiempo libre para compartir y prestar atención a estos, de modo que las madres de la actualidad deben combinar las tareas laborales con las actividades relacionadas con la maternidad, tomándose en cuenta las exigencias individuales y los tiempos disponibles con los que cuenta (Castillo, 2008).

Por último, es importante mencionar el cambio en la concepción de feminidad que vino a dar el lesbianismo, el cual corresponde a una transgresión de las normas de la sexualidad dominante que dotan de ciertas características específicas al género femenino, entre las cuales se destacan la heterosexualidad y la maternidad como obligatorias, (Alfarache, 2003). De manera que una mujer lesbiana no va a cumplir el patrón establecido de ser mujer, pues al no pasar naturalmente por el proceso de embarazo y maternidad, no cumplen con lo que se ha establecido socialmente. Sin embargo, el hecho de no pasar por este proceso no las desvincula del ser mujer y de realizar las actividades, así como de contar con las características que se le han sido otorgadas al género femenino.

\section{Conclusiones}

La maternidad ha sido una característica intrínseca a la feminidad, lo cual se ha visto reflejado a lo largo de toda la historia, constituyéndose esta en un distintivo al cual se apegan las mujeres, además de ser lo esperado socialmente. Es importante rescatar que es la maternidad la cual establece la diferencia entre los géneros, ya que a través de esta se establecen las labores que debe realizar cada género, así como las actitudes y características que se espera que cumplan.

Un aspecto a destacar es como la maternidad en un momento histórico pasó a ser el eje fundamental para la organización social, otorgándosele una mayor importancia de la que se le dio en épocas posteriores. Sin embargo esta posición de la mujer experimentó un cambio, quedando en una posición de inferioridad en relación con el hombre, lo cual se reflejo en la institución de la sociedad patriarcal, manteniendo algunos de esos principios hasta la actualidad.

El proceso de maternidad había sido asociado únicamente con las mujeres, sin embargo a partir del Renacimiento se da la inclusión de la función paterna dentro del proceso, no obstante se debe aclarar que a pesar de este cambio, los deberes y obligaciones que se les atribuyen a ambos sexos son distintos, recayendo sobre las madres el mayor peso. Además, se debe mencionar que esta capacidad de las mujeres de dar vida, ha limitado la manera en que estas viven la sexualidad, teniendo gran influencia en ello las directrices religiosas y sociales que exponen que la vida sexual de la mujer está enfocada en la procreación y no en el disfrute de esta. Sin embargo, se puede decir que en la actualidad se 
ha logrado un cierto cambio con respecto al tema, aunque se mantienen algunas ideas acerca de la represión de la sexualidad femenina.

Asimismo, se debe rescatar como la inserción en el mercado laboral por parte de las mujeres, es uno de los principales cambios que suceden en la concepción de maternidad, pues a partir de este hecho se reconoce la capacidad de la mujer de asumir labores distintas a las que debía realizar en el hogar, incluyendo en este punto el cuido de los niños; para los cuales no poseía el mismo tiempo que antes les otorgaba. Además, cabe señalar que las situaciones generadas a partir del sistema capitalista son las que logran hacer un cambio en como la mujer asumía la maternidad, pues es a través de este que las mujeres se ven en la necesidad de salir de sus hogares a trabajar.

Es importante señalar la labor que ha tenido el movimiento feminista en los cambios de los últimos años, principalmente en la decisión de si se asume la maternidad o no, ya que a través de luchas como la legitimación del aborto, apertura en el uso de anticonceptivos y aceptación de la orientación sexual lésbica, se ha logrado un cambio en la concepción de la maternidad.

Para finalizar, resulta trascendental mencionar cómo el género femenino ha sido transformado a través de los diferentes momentos históricos, sin embargo las características de la maternidad no se han separado de la construcción social que hay alrededor de este género, esto porque a pesar de que se han dado cambios de cómo se vive la maternidad o cómo se asume esta, la concepción de feminidad no se ha desligado de lo referente a ser madre.

\section{Referencias bibliográficas}

Aguirre, A. (1989). Preparación a la maternidad. España: Ediciones Morata. Recuperado de: http://books.google.co.cr/books?id=nznM1 hTBeQC\&pg=P A71\&lpg=PA71\&dq=maternidad+en + la $\%$ \%C3\%A9poca +primitiva\&source $=b \mid$ \&ots=ay3gD4SsK0\&sig=B4wvD5yfWoP9IFvAjSHV3mUEQM0\&hl=es\&sa=X\&e i=fbNsUJS8F-L00gGhs4CQBA\&ved =0CC4Q6AEwAA\#v=onepage \&q=materni dad\%20en\%20la\%20\%C3\%A9poca\%20primitiva\&f=false

Alfarache, A. (2003). Identidades lésbicas y cultura feminista: una investigación antropológica. México: Plaza y Valdés. Recuperado de: http://books.google. co.cr/books? id=9S-k2nNoYyYC\&printsec =frontcover \&dq=lesbianismo $+\mathrm{y}+\mathrm{m}$ aternidad\&hl=es\&sa $=X \& e i=8 e 08 U N n Q K K r w i g K Q k Y H 4 A w \& s q i=2 \& v e d=0 C D U$ Q6AEwAw\#v=onepage\&q=lesbianismo\%20y\%20maternidad\&f=false

Arroyo, L., Montañés, J. y Rechea, C. (1999), Estudios de criminología, volumen 2. España: Universidad de Castilla La Mancha. Recuperado de: http://books. google.co.cr/books?id=zowtsIXeXfUC\&dq=la+Revoluci\%C3\%B3n+Industrial + Finaliza\&hl $=$ es\&source $=$ gbs navlinks $s$ 
Beauvoir, S. (2005). El segundo sexo. México: Editorial catedra.

Blanco, J. y Maya, J. (2005). Fundamentos de Salud Pública. Tomo I. Salud Pública. Colombia: Editorial CIB. Recuperado de: http://books.google.co.cr/books?id $=r M U J L I s P N i I C \& p r i n t s e c=$ frontcover\&hl=es\&source=gbs_ge_summary_r\&c $\mathrm{ad}=0 \# \mathrm{v}=$ onepage $\& \mathrm{q} \& \mathrm{f}=$ false

Castillo, M. (2008). Modelos y prácticas de maternidad: continuidades y cambios en dos generaciones de madres platenses. Revista Mad, 19, 63-79. Recuperado de:

Engels, F. (2001). El origen de la familia, de la propiedad privada y del estado. Del socialismo utópico al socialismo científico. España: Ediciones Mestas.

Everingham, C. (1997). Maternidad: Autonomía y Dependencia: Un estudio desde la psicología. España: Narcea Ediciones. Consultado en: http://books.google.es/books? id=twmBAUNguewC\&printsec=frontcover\&hl=es\&source =g bs ge summary $r \& c a d=0 \# v=$ onepage $\& q \& f=$ false

Gandara, J. y Puigvert, A. (2005). Sexualidad humana: una aproximación integral. Madrid: Editorial Médica Panamericana. Consultado en: http://books. google.co.cr/books?id=gj1ciEAGVEgC\&printsec $=$ frontcover\&hl=es\&source= gbs ge summary $r \& c a d=0 \# v=$ onepage $\& q \& f=$ false

Gonzales, M. (2010). Feminismo, Feminismos: Avances hacia la equidad. En Teorías Psicosociales. (pp.115-152). Costa Rica.

Lewis, J. (1985). Antropología simplificada. México: SELECTOR. Recuperado de: http://books.google.co.cr/books?id=20XWjlE6Mq8C\&printsec =frontco ver\&dq=antropolog $\%$ C $3 \% A D a+$ simplificada\&source $=$ bl\&ots $=$ KWq1GkIjoT \&sig=sPkGS80NjeGCSZbQSiSnAMBhDnw\&hl=es\&sa=X\&ei=oKhsU] 1GYQ8wSX0oDoDw\&ved=0CCsQ6AEwAA

Lozano, M. (2001). La construcción del imaginario de la maternidad en Occidente. Manifestaciones del imaginario sobre la maternidad en los discursos sobre las Nuevas Tecnologías de Reproducción (Tesis de Doctorado) Universidad Autónoma de Barcelona, Valencia, España. Consultado en: http://ddd.uab. cat/pub/tesis/2002/tdx-1107102-120847/mle1de2.pdf

Marrades, L. (2002). Luces y sombras del derecho a la maternidad: Análisis jurídico de su reconocimiento. Valencia: Universidad de Valencia. Recuperado de: http://books.google.co.cr/books?id=UXzDVQJ9IHEC\&printsec=frontcover\&hl $=$ es $\&$ source $=g b s$ ge summary $r \& c a d=0 \# v=$ onepage $\& q \& f=$ false

Molina, M. (2006). Transformaciones Histórico Culturales del concepto de maternidad y sus repercusiones en la identidad de la mujer. Revista Psykhe, 
15(2), 93-103. Recuperado de: http://www.scielo.cl/scielo.php?pid=S0718$\underline{22282006000200009 \& \text { script }=\text { sci arttext }}$

Núñez, P. y Contreras, C. (2002). La licencia por maternidad de la trabajadora. Madrid: Editorial Dykinson. Recuperado de: http://books.google.co.cr/books?

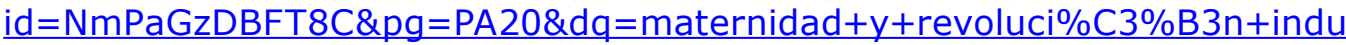
strial\&hl=es\&sa=X\&ei=87G7UIreDYao9gTCzoDIBQ\&ved=0CC8Q6AEwAA\#v $=$ onepage \&q=maternidad $\% 20 y \% 20$ revoluci $\%$ C3\%B3n\%20industrial\&f=false

Romero, M. (1998). La mujer casada y la amazona. Un modelo renacentista en la obra de Pedro Luján. España:Universidad de Sevilla. Recuperado en: http:// books.google.co.cr/books?id=qEmrByjuYEoC\&pg =PA184\&dq=maternidad + en+la+\%C3\%A9poca+del+renacimiento\&hl=es\&sa=X\&ei=E7S7UOHXJI2C 9QSqj4DQCg\&ved =0CDgQ6AEwAg\#v=onepage $\& q=$ maternidad $\% 20$ en $\% 20$ la\%20\%C3\%A9poca\%20del\%20renacimiento\&f=false

Royo, R. (2011). Maternidad, paternidad y conciliación en la CAE: ¿Es el trabajo familiar un trabajo de mujeres? Universidad de Deusto. Recuperado de: http://books.google.co.cr/books?id=XNbFaNqjLgIC\&printsec=frontcover \&hl $=$ es $\&$ source $=g b s$ ge summary $r \& c a d=0 \# v=$ onepage $\& q \& f=$ false

Vélez, A. (2007). Homo Sapiens. Colombia: Villegas Editores, S.A. Recuperado de: http://books.google.co.cr/books?id=cVBYUjOSUooC\&pg=PA504\&lpg=PA $\underline{504 \& d q=\text { maternidad }+e n+l a+v i d a+p r i m i t i v a \& s o u r c e=b l \& o t s=98 Y o N Y g J k C \& s}$ ig=J4WsiSY63QSno5b0ACpbeBwaCoI\&hl=es\&sa=X\&ei=uPJrULOSE4KO8wT7 uYDIAg\&ved=0CC4Q6AEwAA\#v=onepage $\& \mathrm{q}=$ maternidad $\% 20$ en $\% 201 \mathrm{a} \% 20$ vida\%20primitiva\&f=false

Walkowitz, J. (1995). La ciudad de las pasiones terribles: Narraciones sobre peligro sexual en el Londres vitoriano. Valencia: Universitat de València. Recuperado de: http://books.google.co.cr/books?id=y4F9SIH76iMC\&printsec=frontcover $\underline{\& d q}=$ la+ciudad+de+las+pasiones+terribles:+narraciones+sobre+peligro+s exual...\&source=bl\&ots=zecdBGg8LR\&sig=64agDvgaKnpz0zwCDVNGcHW6w $\underline{\mathrm{XY} \& \mathrm{hl}=\mathrm{es} \& \mathrm{sa}=\mathrm{X} \& \mathrm{ei}=\mathrm{Ga9sUM7} \text { Ksuv0AHFrYGwCA\&ved=0CC4Q6AEwAA }}$

Yetano, A. (1988). La Enseñanza Religiosa. En la España de la Restauración (19001920). España: Anthropos Editorial. Recuperado de: http://books.google. co.cr/books?id=IE qp2eDiToC\&pg=PA157\&lpg =PA157\&dq=tareas + de $+\mathrm{la}+\mathrm{m}$ aternidad+en+la+\%C3\%A9poca+primitiva\&source=bl\&ots=WQgJfpnFlq\&sig

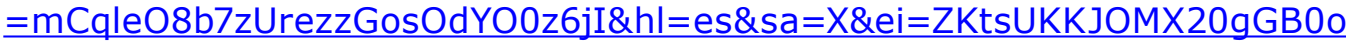
DgCA\&ved=0CDsQ6AEwAg\#v=onepage $\& q=$ tareas $\% 20$ de $\% 201 a \% 20$ maternidad $\% 20$ en $\% 201$ \% $\% 20 \%$ C3\%A9poca $\% 20$ primitiva \&f=false

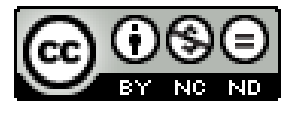

Este obra está bajo una licencia de Creative Commons ReconocimientoNoComercial-SinObraDerivada 4.0 Internacional. 